\title{
Meiotic Gene Segregation in Rhizopus stolonifer
}

\author{
By W. L. GAUGER \\ School of Life Sciences, University of Nebraska-Lincoln, \\ Lincoln, Nebraska 68588 U.S.A.
}

(Received I I February 1977)

\begin{abstract}
Results obtained from the germination of mature zygospores of Rhizopus stolonifer support the hypothesis that normal meiotic processes operate in the sexual cycle of this fungus. The mating-type allele and a second allele, sul, segregated independently. When immature zygospores were germinated no such regularity was observed, but recombinant auxotrophic genotypes were recovered indicating a predormancy meiosis. However, prototrophic recombinants were not found. Bisexual heterokaryotic strains were also recovered from immature zygospore germinations but were auxotrophic. When cultures were obtained from outgrowths of both suspensors of a single zygospore the genotypes were not always those of the respective parents. This suggests the occurrence of nuclear movement from the immature zygospore to the suspensor cells.
\end{abstract}

\section{INTRODUCTION}

Rhizopus stolonifer (Ehrenb. ex Fr.) Vuill. (=R. nigricans) is a heterothallic fungus classified in the Mucorales. Strains of opposite mating type can be crossed to produce the sexual stage, a zygospore. Mating strains are referred to as either $(+)$ or $(-)$. The zygospore is a large (50 to $100 \mu \mathrm{m}$ diam.) thick-walled cell which must undergo a period of dormancy (minimum 30 days) prior to germination. Zygospore germination proceeds with the production of a germsporangium which resembles the vegetative sporangium. Germsporangiospores are formed within the germsporangium. The life cycle of this fungus resembles, in its essential details, that of the better known Phycomyces blakesleeanus.

Although the initial description by Blakeslee (1904) of heterothallism in fungi utilized $R$. stolonifer, the mucoraceous fungi have not, in general, proven amenable to genetic analysis. Recently, reports by Eslava et al. (1975), Eslava, Alvarez \& Delbrück (1975) and Cerdá-Olmedo (1975) have shown that genetic analysis of $P$. blakesleeanus is not only possible but that the results can be interpreted in Mendelian terms. These studies have confirmed genetically some commonly held notions concerning the nuclear events surrounding zygospore formation and germination. The germspores, enclosed in a germsporangium arising from the zygospore, contain mitotic descendents of one or more meiotic products. Furthermore, germspores are usually homokaryotic (Cerdá-Olmedo, I975) and a single germsporangium may contain nuclei derived from more than one meiotic event. A single germsporangium may not contain representatives of all meiotic products. However, pooled data from a number of germinated zygospores provided evidence of Mendelian segregation, i.e. I : I ratios of parental alleles, I : I ratios of parental: recombinant genotypes, etc. It was also concluded that there was no contribution of apogamic nuclei to the germspores (Cerdá-Olmedo, I975).

The initial objective of this study was to elucidate patterns of inheritance in $R$. stolonifer using two-factor crosses. As the work progressed a number of side issues were also investigated. Chief among these was the discovery, based on a report by Hocking (1967) concerning 
$P$. blakesleeanus, that immature zygospores could be induced to undergo precocious germination.

\section{METHODS}

Media. The complete medium (COM) of Minami \& Ikeda (1962) was used at half-strength for routine growth and maintenance of cultures. The minimal medium (SM) contained $\left(\mathrm{g}^{-1}\right)$; D-glucose, 20; DLasparagine, 2.0; $\mathrm{KH}_{2} \mathrm{PO}_{4}, 0.5$; and $\mathrm{MgSO}_{4} \cdot 7 \mathrm{H}_{2} \mathrm{O}$, 0.25. Medium SMCY was SM supplemented with

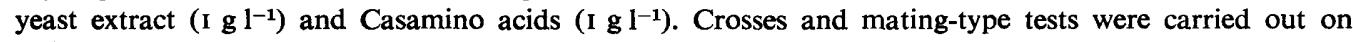
$2 \%(w / v)$ malt extract agar (MEA).

Media were solidified, when necessary, with $1 \cdot 5 \%(w / v)$ Difco Bacto-agar; for SM, washed agar was used. Glass-distilled water was used in the preparation of all defined media. For inhibition of radial expansion of colonies, Triton X-100 $\left(0.24 \mathrm{~g}^{-1}\right)$ was added.

Strains. Wild-type strains UNBI (+) and UNB2 (-), maintained in the culture collection of the School of Life Sciences, University of Nebraska-Lincoln, Nebraska, U.S.A., served as parents as well as mating-type testers. Auxotrophic parents were all descended from a single mutant strain, UNB226B-I 2, and had passed through at least three backcrosses to wild-type parents before being used in crosses. Strain UNB226B-I 2 was isolated after mutagenic treatment of UNBI $(+)$ with $N$-methyl- $N^{\prime}$-nitro- $N$-nitrosoguanidine. Auxotrophic strains did not grow on SM medium but required an additional sulphur source; methionine was routinely used, but cysteine, homocysteine or sodium thiosulphate could be substituted. The mutant is designated $s u l^{-}$. Wild-type strains were prototrophic, growing on unsupplemented SM medium.

Zygospore collection and germination. Mature zygospores produced on MEA were removed, along with vegetative mycelium and spores, and blended for about I min in a Waring Blendor. Brief centrifugation and rinsing repeated eight to ten times gave a relatively clean suspension of zygospores. The zygospores were then spread on an MEA plate and incubated overnight to allow germination of any remaining vegetative spores. Clean zygospores were removed from the plate using a suction apparatus consisting of a finelydrawn glass tip, a large centrifuge tube and a vacuum pump. Under suction the zygospores were drawn into the glass tip and collected in the centrifuge tube. Sterile water was drawn through the apparatus periodically in order to flush collected zygospores into the centrifuge tube.

The zygospores were then dropped, using a Pasteur pipette, on to the surface of a water agar plate. Plugs of agar with attached zygospores were cut out and transferred to a filter disc held on top of a piece of folded filter paper in a shell vial. The vial was capped with a Morton stainless steel closure. Care was taken to keep the zygospores moist. Vials were incubated at 18 to $22{ }^{\circ} \mathrm{C}$.

Germinating zygospores were removed singly to a COM plate, and the germspores spread over the surface. After 8 to 1o $\mathrm{h}$ incubation the germspores had germinated and they were transferred singly to COM slants. Such cultures are referred to as single germspore (SGS) cultures. In most cases ro SGS cultures were sampled from each zygospore germination. The average number of germspores per germsporangium was 350 .

Mating-type tests. All cultures were contrasted separately against both UNBI (+) and UNB2 (-) on MEA.

Test for auxotrophy. Vegetative spores of strains to be tested were inoculated into SM liquid medium. If no growth was detected after $48 \mathrm{~h}$ the strain was considered to be auxotrophic. The response of auxotrophs to methionine $\left(0.2 \mathrm{mg} \mathrm{ml}^{-1}\right)$ was also noted.

Immature zygospore germination. Young zygospores (from a culture 3 to 4 days old) which had not yet developed a thick dark exospore were removed from the culture with fine forceps. They were placed on an MEA plate and adhering mycelium, if present, was teased away. The immature zygospores were observed over the next 6 to $12 \mathrm{~h}$ for evidence of hyphae originating from the zygospore itself. Hyphal tips were then removed and transferred singly to COM slants. It was also possible to detect and remove hyphae originating from the suspensor cells. Hyphal tip cultures obtained from both suspensors of a single zygospore are referred to as paired suspensor isolates.

Reversion experiments. Spore suspensions of auxotrophic strains were spread on the surface of SMCYT plates (SMCY with Triton X-IOO) to estimate the total number of viable spores. A lower dilution of the same spore suspension was spread on SMT plates (SM with Triton X-100). The number of colonies appearing on the latter medium provided an estimate of the reversion frequency.

Scoring. In a two-factor cross, four possible genotypic combinations among the progeny are possible. The germspores in a single germsporangium may be of one type only, or they may represent two, three or all four types. For a single zygospore germination two types of scoring may be employed: (i) the genotype of each SGS culture can be determined and scored; (ii) the presence or absence of each genotype from a single germsporangium can be determined. In the work reported here both types of scoring were used. Eslava et al. (1975) considered that the scoring procedure of noting only the presence or absence of a 
genotype from a germination reduced... 'the effects of the secondary mechanisms causing the disproportions'.

\section{RESULTS}

Two-factor crosses between UNBI (+) and (-) sul ${ }^{-}$strains. A total of 169 mature zygospores from six different crosses were germinated and their products analysed. Single hyphal-tip cultures from 130 immature zygospores representing eight separate crosses were also investigated (Table I, lines I and 2). The results from mature zygospores indicate that the variation from an expected I:I:I:I ratio was significant $(P<0.0 \mathrm{I})$ when the genotypes of all SGS cultures were considered, but was not significant $(P=0.05$ to 0.1$)$ when only the presence or absence of a genotype from each germsporangium was scored.

No such regularity was evident in the results from immature zygospores. Indeed, progeny having one of the two possible recombinant genotypes were recovered, as well as bisexual $(+/-)$ progeny. Bisexual cultures appeared normal and gave no indication of their nature except for the occasional appearance of zygospores in the culture itself. Both the recombinant genotype and the bisexual strains were auxotrophic. In all, auxotrophic strains comprised $85 \%$ of the progeny tested from immature zygospores. Microscopic evidence that hyphae do originate from immature zygospores is presented in Fig. I.

Two-factor crosses between UNB2 (-) and (+) sul- strains. Five crosses involving 159 mature zygospores were analysed; the results are summarized in Table I (lines 3 and 4 ). Again, the variation from the expected ratio was significant $(P<0.01)$ when the genotypes of all progeny were considered, but not $(P=0.1$ to 0.2$)$ when the presence or absence of a genotype from each zygospore was scored. No recombinant progeny were recovered from immature zygospores in 10 crosses involving 106 zygospores, and no bisexual strains were found. The mating-type ratio was disproportionate toward the $(+)$ mating type.

In another cross of this type using a different wild-type parent, UNBII2 (-) sul ${ }^{+}$, some auxotrophic recombinant and bisexual strains were recovered (Table 2).

Segregation ratios for each of the two factors and parental:recombinant ratios are summarized in Table 3. When the mating-type alleles were considered separately, the variation from a 1 : I ratio was significant only when all progeny were considered. For the sul alleles, variation from the expected I : I ratio was significant in the cross $(+) s u l^{-} \times \mathrm{UNB}_{2}(-)$ both for the genotypes of all progeny and for the presence or absence of a genotype from each zygospore. Parental: recombinant ratios were compatible with the hypothesis that the two loci were segregating independently.

One-factor crosses involving only prototrophic strains. The results of three separate crosses with both mature and immature zygospores are summarized in Table I (lines 5 and 6). The data from mature zygospores gave a close fit to the expected $\mathrm{r}: \mathrm{I}$ ratio when the genotypes of all isolates were scored $(P=0.5$ to 0.7$)$ and when each zygospore was scored for the presence or absence of a genotype $(P=0.7$ to 0.8$)$, but the results from immature zygospores were disproportionate for the $(+)$ mating type.

One-factor crosses involving only auxotrophic strains. Surprisingly, $23 \%$ of the progeny from mature zygospores were prototrophic (Table 1 , lines 7 and 8). From immature zygospores, however, the frequency of prototrophs was low $(<\mathrm{I} \%)$. Thirteen percent of the progeny from immature zygospores were bisexual, and, characteristically, auxotrophic. The mating-type ratio (disregarding the bisexual strains) varied significantly from a $\mathrm{I}: \mathrm{I}$ ratio when all isolates were considered $(P<0.05)$ but not significantly when the presence or absence of the type from each zygospore was scored $(P=0.3$ to 0.5$)$.

Reversion frequency. To determine whether or not auxotrophic strains could give rise to prototrophic vegetative progeny, 29 auxotrophic strains were plated at various dilutions on SMCYT and SMT agar plates. Nineteen of the isolates were obtained as single vegetative spore (SVS) cultures from two auxotrophic strains. Ten isolates were derived as SGS cultures from a single zygospore germination. The frequency of appearance of prototrophic 
Table I. Genotypes of progeny recovered from_mature and immature zygospores of Rhizopus stolonifer

The results show the total number of isolates of each genotype and, in parentheses, the total number of zygospores yielding that genotype.

\begin{tabular}{|c|c|c|c|c|c|c|c|c|c|c|c|}
\hline \multirow[b]{3}{*}{ Cross } & \multirow{3}{*}{$\begin{array}{l}\text { No. of } \\
\text { germinated } \\
\text { zygospores }\end{array}$} & \multicolumn{10}{|c|}{ Genotype } \\
\hline & & \multicolumn{2}{|c|}{ (+) $\mathrm{sul}^{+}$} & \multicolumn{2}{|c|}{$(+) \mathrm{sul}^{-}$} & \multicolumn{2}{|c|}{ (-) $s u l^{+}$} & \multicolumn{2}{|c|}{ (-) sul $t^{-}$} & \multicolumn{2}{|c|}{$(+/-) \mathrm{sul}^{-}$} \\
\hline & & No. & $\%$ & No. & $\%$ & No. & $\%$ & No. & $\%$ & No. & $\%$ \\
\hline 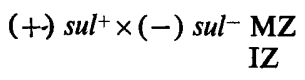 & $\begin{array}{l}169 \\
130\end{array}$ & $\begin{array}{r}347(80) \\
32(29)\end{array}$ & $\begin{array}{l}29(29 \cdot 5) \\
8(\mathrm{I} 5)\end{array}$ & $\begin{array}{l}335(94) \\
169(60)\end{array}$ & $\begin{array}{l}28(30) \\
44(31)\end{array}$ & $\begin{array}{c}257(60) \\
0\end{array}$ & 2I (I9) & $\begin{array}{l}272(80) \\
117(68)\end{array}$ & $\begin{array}{l}22(25 \cdot 5) \\
31(35)\end{array}$ & $\stackrel{0}{64(37)}$ & $17(19)$ \\
\hline${ }_{\mathrm{IZ}}^{(+) \mathrm{sul}^{-} \times(-) \mathrm{sul}^{+} \mathrm{MZ}}$ & $\begin{array}{l}159 \\
106\end{array}$ & $\begin{array}{l}\mathrm{I} 48(57) \\
\mathrm{O}\end{array}$ & $18(23)$ & $\begin{array}{l}315(69) \\
263(90)\end{array}$ & $\begin{array}{l}37(27) \\
88(82)\end{array}$ & $\begin{array}{r}\text { IOI (52) } \\
37(20)\end{array}$ & $\begin{array}{l}12(21) \\
12(18)\end{array}$ & $\begin{array}{l}275(74) \\
0\end{array}$ & $33(29)$ & $\begin{array}{l}0 \\
0\end{array}$ & \\
\hline$(+) \operatorname{sul}^{+} \times(-) \operatorname{sul}^{+} \underset{\mathrm{IZ}}{\mathrm{MZ}}$ & $\begin{array}{r}150 \\
89\end{array}$ & $\begin{array}{l}\text { I59 (97) } \\
289(84)\end{array}$ & $\begin{array}{l}5 \mathrm{I}(5 \mathrm{I}) \\
94(88)\end{array}$ & $\begin{array}{l}\circ \\
\circ\end{array}$ & & $\begin{array}{l}\text { I5I (92) } \\
18 \text { (I2) }\end{array}$ & $\begin{array}{r}49(49) \\
6(12)\end{array}$ & $\begin{array}{l}0 \\
0\end{array}$ & & $\begin{array}{l}\circ \\
\circ\end{array}$ & \\
\hline (+) sul $^{-} \times(-)$sul $^{-}{ }_{\mathrm{IZ}}^{\mathrm{MZ}}$ & $\begin{array}{l}52 \\
8 \mathrm{I}\end{array}$ & $\begin{array}{l}69(\mathrm{I} 4) \\
\mathrm{I}(\mathrm{I})\end{array}$ & $\begin{array}{l}16(17) \\
1(1)\end{array}$ & $\begin{array}{l}219(32) \\
105(44)\end{array}$ & $\begin{array}{l}50(39) \\
50(43)\end{array}$ & $\begin{array}{l}30(8) \\
0\end{array}$ & 7 (10) & $\begin{array}{r}116(25) \\
78(36)\end{array}$ & $\begin{array}{l}27(30) \\
37(36)\end{array}$ & $\begin{aligned} 3 & (3) \\
28 & (20)\end{aligned}$ & $\begin{array}{l}<\mathrm{I}(4) \\
13(20)\end{array}$ \\
\hline
\end{tabular}

MZ, Mature zygospores; IZ, immature zygospores.

Table 2. Genotypes of progeny recovered from the cross $960(5) \mathrm{I}(+)$ sul ${ }^{-} \times \mathrm{UNBII} 2(-)$ sul $^{+}$(immature zygospores only)

The results show the total number of isolates of each genotype and, in parentheses, the total number of zygospores yielding that genotype.

\begin{tabular}{|c|c|c|c|c|c|c|c|c|c|c|}
\hline \multirow{3}{*}{$\begin{array}{l}\text { No. of } \\
\text { germinated } \\
\text { zygospores }\end{array}$} & \multicolumn{10}{|c|}{ Genotype } \\
\hline & \multicolumn{2}{|c|}{ (+) $\mathrm{sul}^{+}$} & \multicolumn{2}{|c|}{${ }^{+}+$sul $^{-}$} & \multicolumn{2}{|c|}{$(-) s u l^{+}$} & \multicolumn{2}{|c|}{ (-) sul- } & \multicolumn{2}{|c|}{$(+/-)$ sul $^{-}$} \\
\hline & No. & $\%$ & No. & $\%$ & No. & $\%$ & No. & $\%$ & No. & $\%$ \\
\hline 23 & 0 & & $16(5)$ & $20(\mathrm{I} 6)$ & $15(9)$ & $19(29)$ & 47 (I6) & $60(52)$ & I (I) & I (3) \\
\hline
\end{tabular}

Table 3. Segregation ratios of progeny from two-factor crosses (mature zygospores only)

The results show the total number of isolates of each type and, in parentheses, the total number of zygospores yielding that type. The variation from the expected I:I ratio is significant at the $5 \%$ probability level for values shown in italics.

Cross
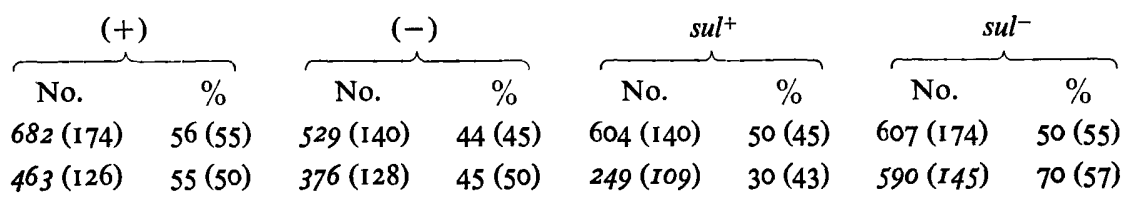

No.
$\left.\begin{array}{cc}619(160) & 51(51) \\ 416(12(1 & 50(48)\end{array}\right)$

(+) $\mathrm{sul}^{+} \times(-) \mathrm{sul}^{-}$

$463(126)$

$55(50)$

$376(128)$

$45(50)$

$\begin{array}{llll}249(109) \quad 30(43) & 590(145) \quad 70(57)\end{array}$

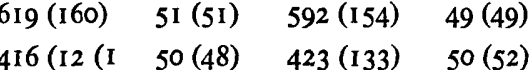




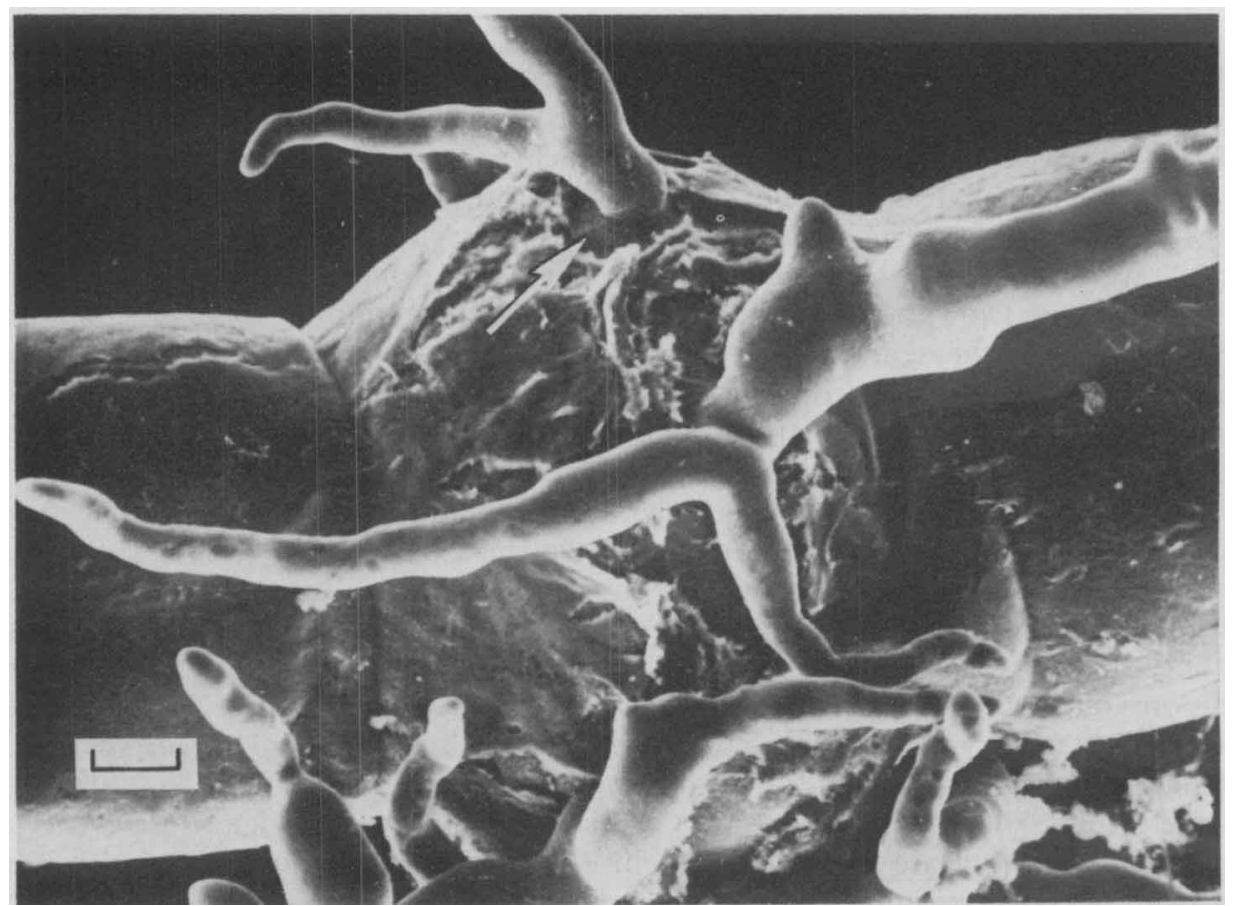

Fig. I. Germinating immature zygospore of Rhizopus stolonifer. The emerging hypha is indicated by the arrow. Material was fixed in $3 \%(\mathrm{w} / \mathrm{v})$ phosphate-buffered glutaraldehyde, postfixed in $\mathbf{I} \%(\mathrm{w} / \mathrm{v})$ osmium tetroxide, and dehydrated in a graded ethanol series before critical point drying with $\mathrm{CO}_{2}$. Specimens were coated with gold/palladium in a sputtering system and examined with a Cambridge Stereoscan S4-10 scanning electron microscope. Bar marker represents Io $\mu \mathrm{m}$.

Table 4. Reversion frequencies of 29 auxotrophic strains

\begin{tabular}{|c|c|c|c|c|}
\hline Parent strain & $\begin{array}{l}\text { Isolates } \\
\text { tested }\end{array}$ & $\begin{array}{l}\text { Total spores } \\
\text { plated }\end{array}$ & $\begin{array}{c}\text { Total } \\
\text { prototrophs }\end{array}$ & $\begin{array}{c}\% \\
\text { Prototrophs }\end{array}$ \\
\hline $\begin{array}{l}1032(5) 2 \\
1032(5) 7 \\
1032(19)^{*}\end{array}$ & $\begin{array}{r}10 \text { (SVS) } \\
9 \text { (SVS) } \\
10 \text { (SGS) }\end{array}$ & $\begin{array}{l}4.98 \times 10^{5} \\
6.05 \times 10^{5} \\
9.64 \times 10^{5}\end{array}$ & $\begin{array}{r}3 I \\
28 \\
4\end{array}$ & $\begin{array}{l}0.00622 \\
0.00462 \\
0.00041\end{array}$ \\
\hline Totals & 29 & $2.07 \times 10^{6}$ & 63 & 0.00304 \\
\hline
\end{tabular}

strains appears to be consistent with a hypothesis of reversion $(3.04$ prototrophs per IO spores) (Table 4).

Recovery of both mating types from bisexual strains. Between 15 and 20 SVS isolates were obtained from each of eight bisexual strains and tested for mating type. Both $(+)$ and $(-)$ isolates were recovered from five of the eight strains; 100 isolates were $(+)$ and 42 were $(-)$. Only one SVS isolate was bisexual.

Paired suspensor isolates. Part of the evidence which Blakeslee (1904) presented to support the hypothesis that $R$. stolonifer was heterothallic was to show that cultures derived from both suspensor cells of a single zygospore were of opposite mating type. Similarly derived cultures from immature zygospore suspensors were tested. The results indicate that while the majority $(5 \mathrm{I} / 68)$ of the paired suspensor isolates tested did represent each of the two parental genotypes, a sizeable number (17/68) did not. In 13 cases, both isolates were of the same parental genotype; in three, they were of the same recombinant genotype; and in one, one isolate was parental while the other was recombinant. 


\section{DISCUSSION}

The results from two-factor crosses utilizing mature zygospores indicate the operation of normal meiotic processes in the sexual cycle of $R$. stolonifer, with independent segregation of the mating type and the sul loci. Although each zygospore germination may not yield representatives of all four meiotic products, nevertheless when the results from large numbers of zygospore germinations are pooled a Mendelian pattern is seen. It must be noted, however, that this is dependent on the method of scoring. If the presence or absence of each genotype from each zygospore is scored, the disproportion observed when total SGS progeny genotypes are considered decreases. These results essentially agree with those presented for $P$. blakesleeanus by Eslava et al. (1975).

Evidence obtained from immature zygospore germination suggests that meiosis must be occurring prior to the onset of zygospore dormancy in $R$. stolonifer. This contradicts the report of Cutter (1942) who concluded, from cytological observations, that meiosis occurred just before zygospore germination at the end of dormancy. It does not preclude the possibility, however, that the germination process itself may bring about the initiation of meiosis. The absence of prototrophic recombinants from immature zygospores is puzzling. Equally difficult to explain is the presence of bisexual strains from immature zygospores only when both nuclear members carry the auxotrophic allele. That the bisexual strains are heterokaryotic is borne out by their segregation, when single vegetative spores are isolated, into component $(+)$ and $(-)$ strains.

If the results from mature zygospores are consistent with normal nuclear events and Mendelian rules, why are the results obtained from immature zygospores inconsistent? How is it that a genotype not found at an early stage is present at a later stage?

A persistent, and disturbing, feature of $s u l^{-} \times s u l^{-}$crosses was the appearance of prototrophic strains among the progeny of mature zygospores: $27 \%$ of the mature zygospores germinated in these crosses yielded some prototrophic SGS cultures and $23 \%$ of the SGS cultures themselves were prototrophic. Reversion rates for the $s^{-}$vegetative cultures were several orders of magnitude lower than this. There are at least three possible explanations for the high frequency of prototrophs. (i) The crosses may have involved nonallelic $\mathrm{sul}^{-}$markers, so that the 'revertants' were really recombinants. The fact that all sul- parents used in these crosses were derived from a single mutant strain detracts from this possibility. (ii) The presence of suppressor mutations could account for the reversion but could not account, by itself, for the large difference observed in reversion frequency between vegetative spores and germsporangiospores. (iii) Reversion, uniquely associated with meiosis, has been reported by Magni \& von Borstel (1962) and Magni (1963). The utilization of the single-celled Saccharomyces cerevisiae allowed them to derive accurate reversion frequencies when comparing vegetative and sporulating cells. The reversion rate following meiosis was six to 20 times higher than that found in vegetative cells (mitotic). The underlying mechanism of reversion was attributed to unequal chromosome exchange during the meiotic process.

Whatever the explanation, it must take into account the likelihood that the reversion process is uniquely associated with the $\mathrm{sul}^{-} \times \mathrm{sul}^{-}$cross. No disproportionate numbers of prototrophs appeared in either of the reciprocal two-factor crosses.

Another unusual feature of the immature zygospore recombinant production was that when a different (-) sul ${ }^{+}$parent in two-factor crosses was used (Table 2), recombinant progeny were recovered and bisexual auxotrophic strains were found. It is thus clear that reciprocal crosses of the sul strains can both produce auxotrophic recombinants as well as bisexual auxotrophic strains, but only when proper combinations of parents are used.

Hawker \& Gooday (I969) have shown in Rhizopus sexualis that the walls separating the suspensor cells from the zygote itself are formed prior to the dissolution of the wall separating the two gametangia. In view of this it is difficult to understand why paired 
suspensor isolates do not always give rise to the respective genotypes of the parents. The results strongly suggest that nuclear movement can occur, following meiosis, from the immature zygospores into the suspensor cells.

Cytological and genetic evidence in P. blakesleeanus (Cerdá-Olmedo, 1975, and personal communication) supports the contention that germspores in that species are predominantly homokaryotic. Vegetative sporangiospores from heterokaryotic cultures may, however, be heterokaryotic (Heisenberg \& Cerdá-Olmedo, 1968). The infrequent occurrence of bisexual SGS cultures in $R$. stolonifer provides marginal evidence that germspores of this species, likewise, are predominantly homokaryotic. Unfortunately, the thick melanized wall of the Rhizopus sporangiospore precludes easy cytological observations.

I would like to acknowledge the technical help of Greg Sorenson, Peter Nwangwu, Joan Tomlinson and Lavonne Hudson. Dr Kit Lee carried out the electron microscopy and the photography. Special thanks are due to the Department of Biochemistry, Marischal College, University of Aberdeen for providing facilities during a leave of absence, and to Dr Graham Gooday for helpful discussions. Financial assistance was provided by the University of Nebraska Research Council and by funds from NIH Biomedical Sciences Support Grant RR-07055-IO.

\section{REFERENCES}

BLAKeSLeE, A. F. (1904). Sexual reproduction in the Mucorineae. Proceedings of the American Academy of Arts and Sciences 40, 205-319.

Cerdi-Olmedo, E. (1975). The genetics of Phycomyces blakesleeanus. Genetical Research 25, 285296.

CUTTER, V. M., JR (1942). Nuclear behavior in the Mucorales. II. The Rhizopus, Phycomyces and Sporodinia patterns. Bulletin of the Torrey Botanical Club 69, 592-616.

Eslava, A. P., Alvarez, M. I., Burke, P. V. \& DELBRÜCK, M. (1975). Genetic recombination in sexual crosses of Phycomyces. Genetics 80, 445462.

Eslava, A. P., Alvarez, M. I. \& Delbrück, M. (1975). Meiosis in Phycomyces. Proceedings of the National Academy of Sciences of the United States of America 72, 4076-4080.

HaWker, L. \& Gooday, M. (I969). Fusion, subsequent swelling and final dissolution of the apical walls of the progametangia of Rhizopus sexualis (Smith) Callen. An electron microscope study. New Phytologist 68, 133-140.

Heisenberg, M. \& Cerdá-Olmedo, E. (1968). Segregation of heterokaryons in the asexual cycle of Phycomyces. Molecular and General Genetics 102, I87-195.

HockING, D. (1967). Zygospore initiation, development and germination in Phycomyces blakesleeanus. Transactions of the British Mycological Society 50, 207-220.

MaGNI, G. E. (1963). The origin of spontaneous mutations during meiosis. Proceedings of the National Academy of Sciences of the United States of America 50, 975-980.

MAGNI, G. E. \& VON BORSTEL, R. C. (1962). Different rates of spontaneous mutation during mitosis and meiosis in yeast. Genetics 47, 1097-1 108 .

Minami, Z. \& IkedA, Y. (1962). Heterokaryosis observed in Rhizopus javanicus. Journal of General and Applied Microbiology 8, 92-98. 